\title{
The Application of Asymmetric Entangled States in Quantum Game
}

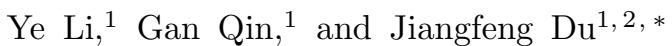 \\ ${ }^{1}$ Hefei National Laboratory for Physical Sciences at Microscale and Department of Modern Physics, \\ University of Science and Technology of China, Hefei 230026, People's Republic of China \\ ${ }^{2}$ Department of Physics, National University of Singapore, Lower Kent Ridge, Singapore 119260, Singapore
}

\begin{abstract}
In the present letter, we propose a more general entangling operator to the quantization of Cournot economic model, in which players can access to a continuous set of strategies. By analyzing the relation between the von Neumann entropy of the entangled state and the total profit of two players precisely, we find that the total profit at the Nash equilibrium always achieves its maximal value as long as the entropy tends to infinity. Moreover, since the asymmetry is introduced in the entangled state, the quantum model shows some kind of "encouraging" and "suppressing" effect in profit functions of different players.
\end{abstract}

PACS numbers: 03.67.-a; 02.50.Le

\section{INTRODUCTION}

Game theory has experienced a great development since early time of last century through its wide application in nearly every aspect of our modern society. In recent few years, along with the thriving of quantum information, a newly-emerging field, quantum game has begun to attract much attention due to the intimate connection between the theory of game and the theory of quantum communication [1]. This field was initiated by a paper of Meyer, finding that a player can always beats his classical opponent by adopting quantum strategies on the coin tossing game 2]. Later, Eisert et al. 1] introduced quantum entanglement into the famous Prisoners' Dilemma, by which the contradiction existing in the classical scenario vanishes. Since then, quantum game theory has developed rapidly and exhibited great superiority over its classical counterpart in later works $3,4,5,6,7,8,9,10,11,12,13$. In addition to theoretical investigations, $\mathrm{Du}$ et al. realized the quantum Prisoners' Dilemma in experiments for the first time 14], and therefore constructed a practical base for quantum game.

However, most of previous investigations on quantum game mainly focused on game models with discrete strategies set. In Li et al.'s paper 16], they first put forward a quantization scheme for Cournot duopoly, a famous economical model even preceding the birth of game theory [15], in which two players can virtually cooperate and get the optimal profit at the maximal quantum entanglement between them albeit both act "selfishly" just as they do in the classical case. This work actually set up a foundation for further discussions regarding quantum game with continuous strategies [17, 18, 19]. Recently Qin et al. introduced a quantization scheme for asymmetric games 20]. The key modification of their scheme compared with Li et al.'s is the adoption of two differ-

*Electronic address: djf@ustc.edu.cn ent entanglement factors, which ensure the asymmetric games to get the optimal cooperative profit. Nevertheless, Qin et al.'s quantum scheme can still be regarded as a fair play, for the two factors are determined by the extent of the asymmetry of the game. It is quite often that various environments may affect the quantum game, leading to asymmetric forms of the entanglement operator.

Hence, to better understand the property of quantum game, it's worthwhile studying the distinctive features of quantum game resulted from the change of the entanglement operator. In this letter we develop the quantum model of Cournot's duopoly proposed by Li et al. by changing the initial state before actually entangling them, and we define these operations altogether as a whole entangling operation. Thus it can be discovered that the total profit at the unique Nash equilibrium will increase monotonously as long as the entropy of the entangled state, namely the entanglement, tends to infinity. While, considering the lack of symmetry in this quantization scheme, our result will not remain Pareto optimal (the best result that can be achieved without disadvantaging at least one group), which in fact may not be a necessity in the real world because of the simple fact that there is no absolutely-impartial law. Besides, all of the operators we cite in the quantization of Cournot duopoly can be realized experimentally within the capacity of modern optical technology through proper design.

\section{COURNOT DUOPOLY}

Now we briefly recall the classical Cournot duopoly. Duopoly is an economic condition in which two firms hold the market of a certain commodity without a third competitor. In Cournot model, two firms are assumed to produce a homogeneous product and simultaneously decide their quantities $q_{1}$ and $q_{2}$. and the price of this 


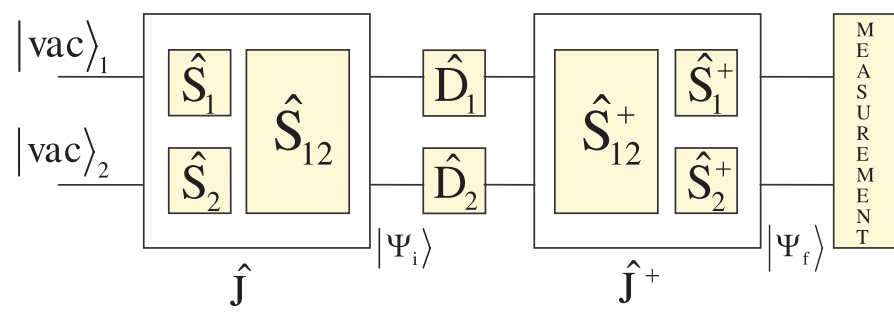

FIG. 1: The quantum structure of the game.

product is decided by the quantities as

$$
p=\left\{\begin{array}{cc}
a-Q & (Q<a) \\
0 & (Q \geq a)
\end{array}\right.
$$

where $Q$ is the total quantity of two firms, i.e. $Q=q_{1}+q_{2}$ and $a$ is a constant. Assume that the unit cost of the product is also a constant $c$, the profit function of each firm would be:

$$
U_{j}=q_{j}(p-c)=q_{j}\left(k-\left(q_{1}+q_{2}\right)\right) \text {, }
$$

with $k=a-c>0$ and $j=1,2$. It's easy to work out the unique Nash equilibrium:

$$
\begin{aligned}
& q_{1}^{*}=q_{2}^{*}=\frac{k}{3}, \\
& u_{1}^{*}=u_{2}^{*}=\frac{k^{2}}{9} .
\end{aligned}
$$

However, the Nash equilibrium is not the optimal solution for the market. If the two firms cooperate and raise their total quantity to $k / 2$, they can acquire the highest total profit $k^{2} / 4$. In addition, by keeping the symmetry of the model, we can obtain the Pareto optimal in the following solution:

$$
\begin{aligned}
& q_{1}^{*}=q_{2}^{*}=\frac{k}{4}, \\
& u_{1}^{*}=u_{2}^{*}=\frac{k^{2}}{8} .
\end{aligned}
$$

The profit difference between the optimal choice and the Nash equilibrium reflects the conflict between individual rationality and collective rationality, just like in the classical prisoners' dilemma.

\section{QUANTIZATION OF CLASSICAL MODEL}

Here we put forward a modified quantization model of $\mathrm{Li}$ et al.'s [16], where we make use of two singlemode electromagnetic fields. Fig. 1 1 shows the quantum structure of the game. As a extension, we first apply two single-mode squeezing operator $\hat{S}_{1} \otimes \hat{S}$ to the vacuum state, where $\hat{S}_{j}=\exp \left(\frac{\gamma_{j}}{2} a_{j}^{2}-\frac{\gamma_{j}}{2} a_{j}^{\dagger} 2\right)$, and $a_{j}^{\dagger}, a_{j}$ are the creation and annihilation operator of the corresponding field, followed by a two-mode squeezing operator $\hat{S}_{12}=\exp \left(\gamma a_{1} a_{2}-\gamma a_{1}^{\dagger} a_{2}^{\dagger}\right)$ of a two-mode electromagnetic field. Thus the total entanglement operator would be:

$$
\begin{aligned}
\hat{J}\left(\gamma_{1}, \gamma_{2}, \gamma_{12}\right) & =\hat{S}_{12} \hat{S}_{1} \hat{S}_{2} \\
& =\exp \left[\gamma_{12}\left(a_{1} a_{2}-a_{1}^{\dagger} a_{2}^{\dagger}\right)\right] \\
& \cdot \exp \left[\gamma_{1}\left(a_{1}{ }^{2}-a_{1}^{\dagger}\right) / 2\right] \\
& \cdot \exp \left[\gamma_{2}\left(a_{2}{ }^{2}-a_{2}^{\dagger 2}\right) / 2\right],
\end{aligned}
$$

with constraint $\gamma_{1}, \gamma_{2}, \gamma_{12} \in R$ and $\gamma_{12} \geq 0$ (which also is an extension of Li et al's model). Apparently, we can turn back to Li et al's entanglement operator if $\gamma_{1}=\gamma_{2}=$ 0 . The strategies in the set of local unitary operators is $\hat{D}_{j}\left(x_{j}\right)=\exp \left[x_{j}\left(a_{j}^{\dagger}-a_{j}\right) / \sqrt{2}\right]$ as the scheme of Li et al.'s.

The final measurement is made corresponding to the observables $\hat{X}_{j}=\left(a_{j}^{\dagger}+a_{j}\right) / \sqrt{2}$, which in quantum optics is the amplitude quadrature (we can simply perceive it as the "position" operator). This measurement is done by the homodyne measurement assuming that the state is infinitely squeezed. Correspondingly, by citing the phase quadrature $\hat{P}_{j}=i\left(a_{j}^{\dagger}-a_{j}\right) / \sqrt{2}$ (which can be seen as the "momentum" operator), we can rewrite the entanglement operator and the strategies in the following form:

$$
\begin{aligned}
\hat{J}\left(\gamma_{1}, \gamma_{2}, \gamma_{12}\right)= & \exp \left[i \gamma_{12}\left(\hat{X}_{1} \hat{P}_{2}+\hat{X}_{2} \hat{P}_{1}\right)\right] \\
& \cdot \exp \left[i \gamma_{1}\left(\hat{X}_{1} \hat{P}_{1}+\hat{P}_{1} \hat{X}_{1}\right) / 2\right] \\
& \cdot \exp \left[i \gamma_{2}\left(\hat{X}_{2} \hat{P}_{2}+\hat{P}_{2} \hat{X}_{2}\right) / 2\right], \\
\hat{D}_{j}\left(x_{j}\right)= & \exp \left(-i x_{j} \hat{P}_{j}\right), \quad j=1,2 .
\end{aligned}
$$

Obviously, the classical Cournot duopoly is only a special case with $\gamma_{12}=0$ in its quantum extension. Because the strategy set is not extended compared to its classical counterpart, we can be sure that all the features shown in the quantization version attribute to the entanglement of the states. The specific property of the entangled state is analyzed in the paragraphs below.

By detailed calculation we get:

$\hat{J}^{\dagger} \hat{D}_{1}\left(x_{1}\right) \hat{J}=\exp \left[-i x_{1}\left(\hat{P}_{1} e^{\gamma_{1}} \cosh \gamma_{12}+\hat{P}_{2} e^{\gamma_{2}} \sinh \gamma_{12}\right)\right]$

$\hat{J}^{\dagger} \hat{D}_{2}\left(x_{2}\right) \hat{J}=\exp \left[-i x_{2}\left(\hat{P}_{2} e^{\gamma_{2}} \cosh \gamma_{12}+\hat{P}_{1} e^{\gamma_{1}} \sinh \gamma_{12}\right)\right]$.

Therefore the final state reads:

$$
\begin{aligned}
\left|\psi_{f}\right\rangle & =\hat{J}^{\dagger}\left(\hat{D}_{1} \otimes \hat{D}_{2}\right)\left|\psi_{i}\right\rangle \\
& =\exp \left[-i e^{\gamma_{1}}\left(x_{1} \cosh \gamma_{12}+x_{2} \sinh \gamma_{12}\right) \hat{P}_{1}\right]|v a c\rangle_{1} \\
& \otimes \exp \left[-i e^{\gamma_{2}}\left(x_{2} \cosh \gamma_{12}+x_{1} \sinh \gamma_{12}\right) \hat{P}_{2}\right]|v a c\rangle_{2}
\end{aligned}
$$


And the measurement gives the respective quantity of each firm player:

$$
\begin{aligned}
& q_{1}=e^{\gamma_{1}}\left(x_{1} \cosh \gamma_{12}+x_{2} \sinh \gamma_{12}\right), \\
& q_{2}=e^{\gamma_{2}}\left(x_{2} \cosh \gamma_{12}+x_{1} \sinh \gamma_{12}\right)
\end{aligned}
$$

Simply by substituting the above into the classical profit functions (see Eq. (2)), we get the quantum profit:

$$
\begin{aligned}
& u_{1}^{Q}\left(x_{1}, x_{2}\right)=e^{\gamma_{1}}\left(x_{1} \cosh \gamma_{12}+x_{2} \sinh \gamma_{12}\right) p\left(x_{1}, x_{2}\right) \\
& u_{2}^{Q}\left(x_{1}, x_{2}\right)=e^{\gamma_{2}}\left(x_{2} \cosh \gamma_{12}+x_{1} \sinh \gamma_{12}\right) p\left(x_{1}, x_{2}\right)
\end{aligned}
$$

where $p\left(x_{1}, x_{2}\right)=\left[k-x_{1}\left(e^{\gamma_{1}} \cosh \gamma_{12}+e^{\gamma_{2}} \sinh \gamma_{12}\right)-\right.$ $\left.x_{2}\left(e^{\gamma_{2}} \cosh \gamma_{12}+e^{\gamma_{1}} \sinh \gamma_{12}\right)\right]$.

Solving the Nash equilibrium equations we obtain the unique solution:

$$
\begin{aligned}
& x_{1}^{*}=\frac{k e^{\gamma_{2}} \cosh \gamma_{12}}{\left(e^{\gamma_{1}}+e^{\gamma_{2}}\right) \sinh 2 \gamma_{12}+e^{\gamma_{1}+\gamma_{2}}\left(2 \cosh 2 \gamma_{12}+1\right)}, \\
& x_{2}^{*}=\frac{k e^{\gamma_{1}} \cosh \gamma_{12}}{\left(e^{\gamma_{1}}+e^{\gamma_{2}}\right) \sinh 2 \gamma_{12}+e^{\gamma_{1}+\gamma_{2}}\left(2 \cosh 2 \gamma_{12}+1\right)} .
\end{aligned}
$$

And the profit at the equilibrium reads:

$$
\begin{aligned}
& u_{1}^{Q}\left(x_{1}^{*}, x_{2}^{*}\right)=\frac{\cosh \gamma_{12}\left(\cosh \gamma_{12}+e^{\Delta \gamma} \sinh \gamma_{12}\right)^{2}\left(\cosh \gamma_{12}+e^{-\Delta \gamma} \sinh \gamma_{12}\right)}{\left(1+2 \cosh 2 \gamma_{12}+2 \cosh \Delta \gamma \sinh 2 \gamma_{12}\right)^{2}} k^{2}, \\
& u_{2}^{Q}\left(x_{1}^{*}, x_{2}^{*}\right)=\frac{\cosh \gamma_{12}\left(\cosh \gamma_{12}+e^{-\Delta \gamma} \sinh \gamma_{12}\right)^{2}\left(\cosh \gamma_{12}+e^{\Delta \gamma} \sinh \gamma_{12}\right)}{\left(1+2 \cosh 2 \gamma_{12}+2 \cosh \Delta \gamma \sinh 2 \gamma_{12}\right)^{2}} k^{2},
\end{aligned}
$$

where $\Delta \gamma=\gamma_{1}-\gamma_{2}$. Apparently, the profit functions only depend on two parameters $\gamma_{12}$ and $\Delta \gamma$. In addition, if we make $\gamma_{1}=\gamma_{2}=0$, i.e. $\Delta \gamma=0$, it's easy to find that the result is exactly that of $\mathrm{Li}$ et al in their quantization scheme.

\section{ENTROPY \& ASYMMETRY}

Using the method put forward in Rendell's paper 21], we write the entangled state in the representation of "position":

$$
\left|\psi_{i}\right\rangle=\exp \left[-\left(\alpha x_{1}^{2}+\beta x_{2}^{2}+2 \gamma x_{1} x_{2}\right) / 2\right] .
$$

By solving the equations below:

$$
\begin{aligned}
\hat{J} a_{1} \hat{J}^{\dagger}\left|\psi_{i}\right\rangle & =\hat{J} a_{1} \hat{J}^{\dagger} \hat{J}|v a c\rangle_{1}=0, \\
\hat{J} a_{2} \hat{J}^{\dagger}\left|\psi_{i}\right\rangle & =\hat{J} a_{2} \hat{J}^{\dagger} \hat{J}|v a c\rangle_{2}=0 .
\end{aligned}
$$

We can obtain the expression of the three unknown parameters as

$$
\begin{aligned}
& \alpha=\frac{\left(1+\lambda_{1}\right)\left(1-\lambda_{2}\right)+\left(1-\lambda_{1}\right)\left(1+\lambda_{2}\right) \lambda_{12}^{2}}{\left(1-\lambda_{1}\right)\left(1-\lambda_{2}\right)\left(1-\lambda_{12}^{2}\right)}, \\
& \beta=\frac{\left(1-\lambda_{1}\right)\left(1+\lambda_{2}\right)+\left(1+\lambda_{1}\right)\left(1-\lambda_{2}\right) \lambda_{12}^{2}}{\left(1-\lambda_{1}\right)\left(1-\lambda_{2}\right)\left(1-\lambda_{12}^{2}\right)}, \\
& \gamma=\frac{2 \lambda_{12}\left(1-\lambda_{1} \lambda_{2}\right)}{\left(1-\lambda_{1}\right)\left(1-\lambda_{2}\right)\left(1-\lambda_{12}^{2}\right)}
\end{aligned}
$$

where $\lambda_{1}=\tanh \gamma_{1}, \lambda_{2}=\tanh \gamma_{2}, \lambda_{12}=\tanh \gamma_{12}$. To better assess the entanglement of the state, we need to calculate the von Neumann entropy of the state, which is defined as $S=-\operatorname{Tr}_{1}\left(\rho_{1} \ln \rho_{1}\right)=-\operatorname{Tr}_{2}\left(\rho_{2} \ln \rho_{2}\right)\left(\rho_{1}, \rho_{2}\right.$ are the density matrix of two single-mode electromagnetic fields). Based on the above calculation we get the following equality by making use of Rendell's method:

$$
S(\eta)=\ln (\eta / 2)+\frac{1}{2} \sqrt{\eta^{2}+1} \ln \frac{\sqrt{\eta^{2}+1}+1}{\sqrt{\eta^{2}+1}-1},
$$

where $\eta=\sinh 2 \gamma_{12} \cosh \Delta \gamma \geq 0$. As a confirmation, when $\gamma_{1}=\gamma_{2}=0$, Eq. (25) is reduced to:

$$
S=\cosh ^{2} \gamma_{12} \ln \left(\cosh ^{2} \gamma_{12}\right)-\sinh ^{2} \gamma_{12} \ln \left(\sinh ^{2} \gamma_{12}\right),
$$

which is exactly the entropy of a two-mode squeezing state. It is interesting to find that like the profit function, the entropy is also determined by only two parameters $\gamma_{12}$ and $\Delta \gamma$. In addition, we find that the von Neumann entropy monotonously increases from zero to infinity as the parameter $\eta$ increases. Since $\eta$ increases as either of the two parameters increases (when $\gamma_{12} \neq 0$ ), the entropy is an increasing function of both $\gamma_{12}$ and $\Delta \gamma$.

In Li et al.'s entanglement operator, through whole unitary transformation $\exp \left[\gamma_{12}\left(a_{1} a_{2}-a_{1}^{\dagger} a_{2}^{\dagger}\right)\right]$, we get the maximal entangled state as $\gamma_{12}$ tends to infinity. Nevertheless, it's discovered that only by changing the initial state via local unitary transformation $\exp \left[\gamma_{i}\left(a_{i}{ }^{2}-\right.\right.$ $\left.\left.a_{i}^{\dagger 2}\right) / 2\right]$, the maximized entanglement is also achieved as 
long as entanglement exists (i.e. $\gamma_{12} \neq 0$, even if it is very small; see Fig. (3). Obviously, such kind of situation is impossible in quantum game with Hilbert space of finite dimensions.

Moreover, we estimate the asymmetry by calculating the relative difference of the "position" uncertainties (which actually represent the width of Gaussian wave package in each "position" space) between two parties in the expression below:

$$
\frac{\left\langle\Delta x_{1}^{2}\right\rangle-\left\langle\Delta x_{2}^{2}\right\rangle}{\left\langle\Delta x_{1}^{2}\right\rangle+\left\langle\Delta x_{2}^{2}\right\rangle}=-\frac{\tanh \Delta \gamma}{\cosh 2 \gamma_{12}}
$$

For fixed and finite $\gamma_{12}$, the above expression would monotonously decreases as $\Delta \gamma$ increases. Due to the obvious fact that the system is completely symmetric when $\Delta \gamma=0$, we can simply perceive $|\Delta \gamma|$ as a measurement of the asymmetry of the entangled state, while it's worth mentioning that all the discussion concerning asymmetry above only reflects a variation trend between variables and the final state.

\section{DISCUSSION}

So far, we have confined three parameters presented in the entanglement operator to only two ones, i.e. $\gamma_{12}, \Delta \gamma$. In the paragraphs below, specific analysis of the relation between the game model adopted here and the entangled state is given.

Since the total profit of two players cannot achieve the maximum at the Nash equilibrium due to the deviation between individual rational choice and collective optimization requirement in classical Cournot duopoly, it must be the application of quantum entanglement that eliminates this dilemma in $\mathrm{Li}$ et al.'s scheme. As an extension of their entangled state, we separately give the alternation trends of the total profit $\left(u=u_{1}+u_{2}\right)$ and the entropy $S$ versus $\gamma_{12}, \Delta \gamma$ in the following figures (Fig. 2. Fig. 3), in which the unit of profit is taken as $k^{2} / 4$, namely, the optimal value of the total profit in the classical cooperation scenario (See Eq. (4)).

It's obvious to see that both the profit and entropy function increases as either of the parameters increases (the condition $\gamma_{12} \neq 0$ is necessary here; otherwise there would be no entanglement between two parties). When the entropy tends to infinity, the total profit also achieves its maximal value, and $\mathrm{Li}$ et al.'s situation is just a special case when $\Delta \gamma=0$. Since the entropy is defined as a measurement of the entanglement of the state, it would be adequate to explain that the entanglement of the state does help improve the total profit in Cournot duopoly independent of the concrete form of the state (i.e. no matter in which way the entanglement is increasing) as illustrated in the previous section, and so long as the maximal entanglement is attained, the conflict between individual rationality and collective rationality disappears.
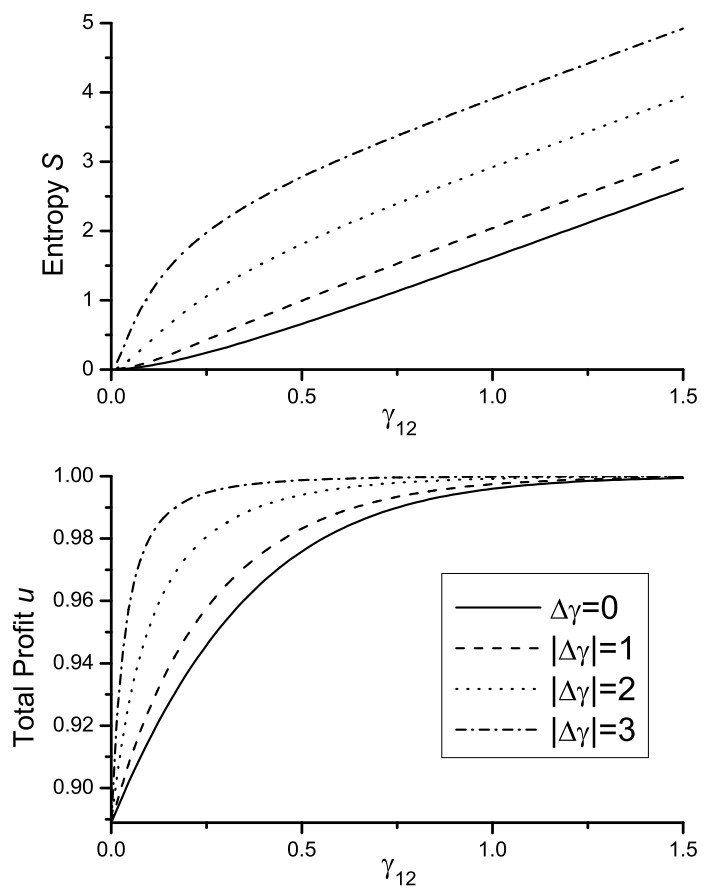

FIG. 2: when $\Delta \gamma$ is fixed, the total profit $u$ compares to the entropy $S$ as $\gamma_{12}$ changes.
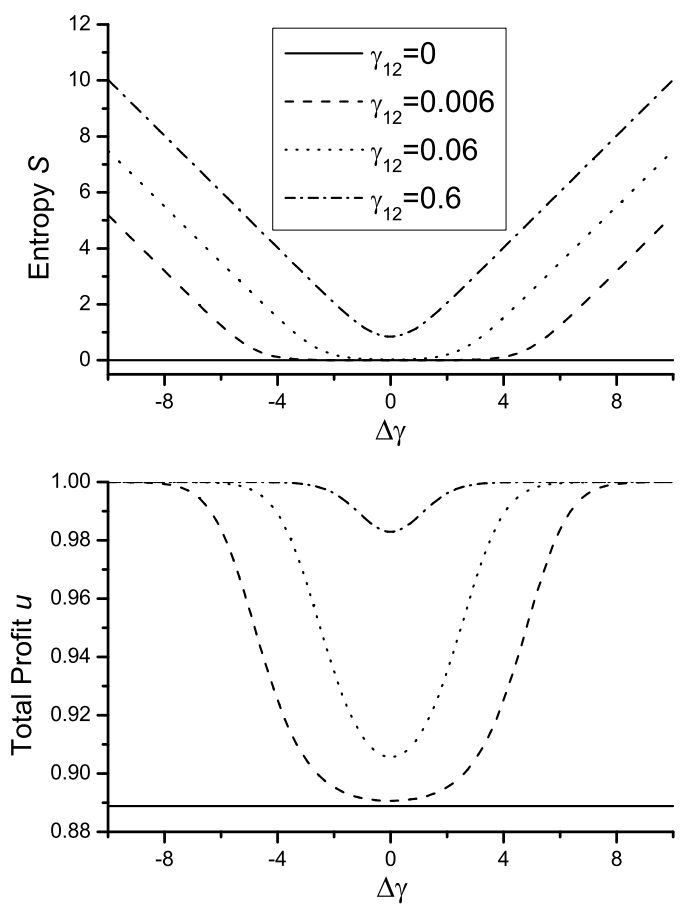

FIG. 3: when $\gamma_{12}$ is fixed, the total profit $u$ compares to the entropy $S$ as $\Delta \gamma$ changes. 


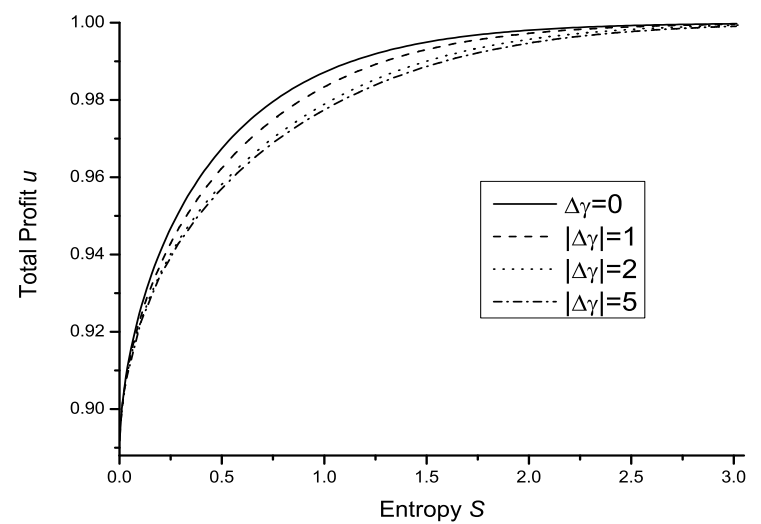

FIG. 4: when $\Delta \gamma$ is fixed, the function plot between the entropy $S$ and total profit $u$.

In Fig. 4 we present a more detailed function plot between the entropy and the total profit. Apparently, although both quantities have the same increasing trend as seen in Fig. [2] and Fig. [3] the entropy does not determine the total profit uniquely, i.e. there isn't a one-to-one correspondence between such two quantities. In combination of previous discussion concerning asymmetry, it is not difficult to find that the increase of asymmetry would cause the reducing of the total profit if the entanglement of the state don't reach the maximum.

Furthermore, the variation of asymmetry also results in the change of the profit difference between two players in our quantization scheme. Fig. 5 shows that Player 1 would monopolize the market and get the optimal profit when $\Delta \gamma$ tends to positive infinity, while on the contrary, player 2 would become the monopolizer of the market if $\Delta \gamma$ tends to negative infinity. Therefore, $\gamma_{1}, \gamma_{2}$ can be separately regarded as the extent to which player 1 and 2 are encouraged (or the corresponding opponents are suppressed), that is, the larger the parameter is, the more profit the corresponding player can acquire.

\section{CONCLUSION}

We investigate the quantization of games with continuum strategic space by making use of a more general entanglement operator. For the particular case of symmetric scenario, it would turn back to the quantization scheme put forward by $\mathrm{Li}$ et al.. As an extension of their quantum model, we investigate the relation between the Nash equilibrium in quantum situation and the entanglement of the state more specifically, and find that the optimal total profit can always be attained as long as the maximal entanglement is realized. Also we observed some novel features such as the encouraging and suppressing effect in our quantum model, which completely

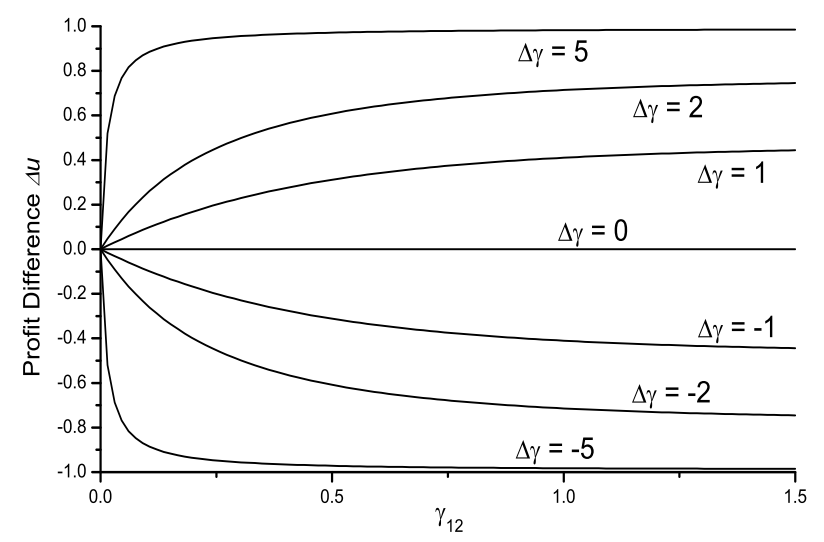

FIG. 5: the variation trend of the profit difference as parameters change.

attributes to the asymmetry of the system. Therefore, in our quantization scheme, the government would have more choices in managing the market. It actually constitutes a better base for further discussion of game models involving incomplete information or unequal competition status.

\section{ACKNOWLEDGEMENTS}

This work is supported by the National fundamental Research Program (Grant No. 2001CB309300), the National Science Fund for Distinguished Young Scholars (Grant No. 10425524) and the Project in Quantum Information Technology (Grant No. R-144-000-071-305).
[1] J. Eisert, M. Wilkens, M. Lewenstein, Phys. Rev. Lett. 83 (1999) 3077.

[2] D.A. Meyer, Phys. Rev. Lett. 82 (1999) 1052.

[3] L. Marinatto, T. Weber, Phys. Lett. A 272 (2000) 291.

[4] N.F. Johnson, Phys. Rev. A 63 (2001) 020302.

[5] W.Y. Hwang, D. Ahn, S.W. Hwang, Phys. Rev. A 64 (2001) 064302.
[6] J.L. Chen, L.C. Kwek, C.H. Oh, Phys. Rev. A 65 (2002) 052320.

[7] A. Iqbal, A.H. Toor, Phys. Rev. A 65 (2002) 022306; Phys. Rev. A 65 (2002) 052328; J. Phys. A: Math. Gen. 37 (2004) 5873.

[8] A.P. Flitney, D. Abbott, Phys. Rev. A 65 (2002) 062318; J. Phys. A: Math. Gen. 38 (2005) 449. 
[9] H. Fort and S. Viola, Phys. Rev. E 69 (2004), 036110.

[10] A. Nawaz and A. H. To, J. Phys. A: Math. Gen. 37 (2004) 4437.

[11] S.K. Oumlzdemir, J. Shimamura, N. Imoto, Phys. Lett. A 325 (2004) 104; Shimamura J, Ozdemir SK, Morikoshi F, Imoto N, Int. J. Quan. Inform. 2 (2004) 79.

[12] E.W. Piotrowski, J. Sladkowski, 2 (2004) 495; Physica A, 345 (2005) 185.

[13] Q. Chen, Y. Wang, J.T. Liu, K.L. Wang, Phys. Lett. A 327 (2004) 98.

[14] J. Du, H. Li, X. Xu, M. Shi, J. Wu, X. Zhou, R. Han, Phys. Rev. Lett. 88 (2002) 137902.

[15] A. Cournot, Researches Into the Mathematical Principles of the Theory of wealth, Macmillan, New York, 1897.

[16] H. Li, J. Du, S. Massar, Phys. Lett. A 306 (2002) 73-78.

[17] J.F. Du, H. Li, C.Y. Ju, Phys. Rev. E 68 (2003) 016124; J.F. Du, H. Li, C.Y. Ju, J. Phys. A: Math. Gen. 38 (2005)
1559.

[18] C.F. Lo, D. Kiang, Phys. Lett. A 318 (2003) 333, Phys. Lett. A 321 (2004) 94, Phys. Lett. A 346 (2005) 65.

[19] G. Qin, X. Chen, M. Sun, J.F. Du, J. Phys. A: Math. Gen. 38 (2005) 4247; X. Chen, G. Qin, X.Y. Zhou, J.F. Du, Chin. Phys. Lett. 22 (2005) 1033.

[20] G. Qin, X. Chen, M. Sun, X.Y. Zhou, J.F. Du, Phys. Lett. A 340 (2005) 78.

[21] R.W. Rendell and A.K. Rajagopal, Phys. Rev. A 72, 012330 (2005).

[22] D.F. Walls and G.J. Milburn, Quantum Optics, SpringerVerlag.

[23] S.L. Braunstein, Review of Modern Physics, 77, 513 (2005).

[24] S.L. Braunstein and H.J. Kimble, Phys. Rev. Lett. 80, 869 (1998). 